\title{
Tidal Enhancement of Internal Structures
}

\author{
Tapan K. Chatterjee \\ Facultad de Ciencias, Fisico-Matematicas, Universidad A. Puebla, A. \\ P. 1316, Puebla, Mexico
}

\section{Introduction and Theory}

Observational evidence indicates that the frequency of internal structures (such as rings, bars, spirals, etc.) is preferentially enhanced for spirals in binary systems (e.g. Elmegreen et al. 1990). In this context we study the tidal effects produced due to two spiral galaxies in a near grazing relative orbit with a near parabolic relative velocity, as such barely bound encounters are the most frequent ones. Both the spirals are endowed with an inner and an outer ring.

The spiral galaxies (of radius $R$ ) are modeled by an exponential disk of scale length $\alpha=4 / R$, with a (static) thickness (Chatterjee 1990), and a spherical polytropic bulge ( $n=0,3,4$ equally weighted combination) containing $1 / 3$ of the mass; about $10 \%$ of the mass of the disk contains gas particles. Two polytropic rings ( $n=1$; Ostriker 1964), are projected on the disk at $1 / 4$ (inner) and $3 / 4$ (outer) of its radius. Though the rings are gaseous, they are here treated as density enhancements. The gravitational potential is softened with softening constants of $\epsilon=r_{o} / 5, r_{o} / 3$, and $0.8 r_{o}$, for the bulge, stellar and gaseous components of the disk, respectively. Here $r_{o}$ is the radius containing $75 \%$ of the total mass of the spiral. The softening constant for the polytropic rings is 0.35 , while the mutual gravitational interaction is softened with a softening constant of $r_{0} / 4$.

The Lagrangian Equations (in polar coordinates $r$ and $\theta$ ) are used to determine the relative orbit of the perturber (Galaxy $\mathrm{p}$ ) with respect to the test system (Galaxy t): $d t=\left[2 \mu^{-1}\left(E-W(r)-\frac{L^{2}}{2 \mu r^{2}}\right)\right]^{-0.5}$, and $d \theta=\frac{L}{\mu r^{2}} d t$, where $\mu, L$ and $E$ are the reduced mass, angular momentum and the orbital energy, respectively, and $W(r)$ is the instantaneous potential energy of interaction between the galaxies. At small intervals of $r$ and $\theta$, the tidal effects and the corresponding changes in velocities of the stars are determined, such that the instantaneous internal energy changes of the two galaxies $\Delta U_{p}(t)$ and $\Delta U_{t}(t)$ can be determined; the instantaneous relative velocity follows from $V(t)=$ $\left[2 \mu^{-1}\left(E_{i}-W(t)-\Delta U_{p}(t)-\Delta U_{t}(t)\right)\right]^{-0.5}, E_{i}$ being the initial value of $E$. The galaxies are interchanged at each interval to determine the relevant quantities for each one (cf. Chatterjee 1992). The three-body approach is adopted to study the motion of the stellar and gaseous particles (but taking the extended nature of the particles into account through gravity softening). All the particles are initially given circularly symmetric motion. (We do not consider the rotation of the galaxies.) 


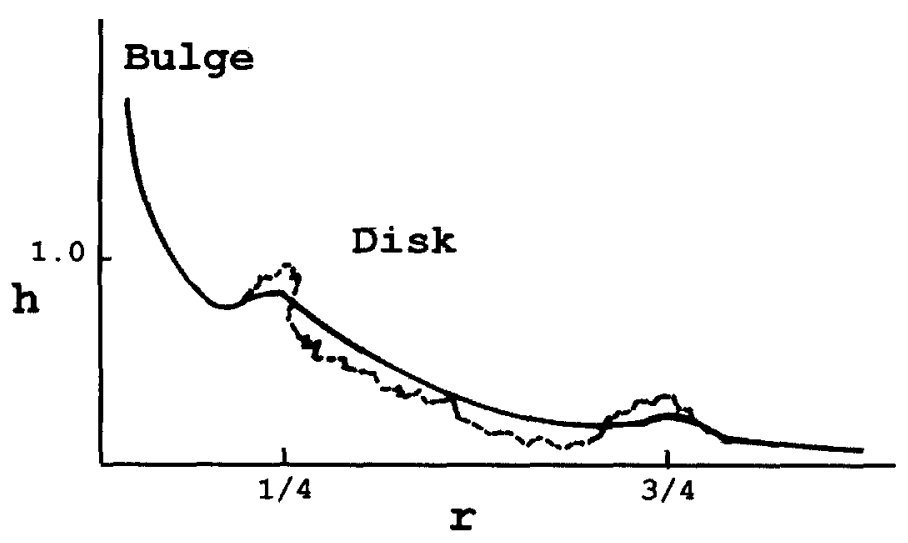

Figure 1. Evolution of the density with radius for the model described in the text. The solid curve is for the initial state, and the dashed curve is for the final state.

\section{Results and Conclusions}

Essentially the external tidal forcing causes the constituents of each galaxy to experience a gravitational torque as the galaxies encounter each other, moving in opposite directions, which acquires a maximum effect shortly after the pericentric approach of the galaxies. The gas (particles), being lighter, is strongly affected by this torque and loses its circularly symmetric motion. However, the effect is most marked in the outer regions of the two galaxies, and the gas interior to the inner ring is hardly affected. Essentially the two rings are density enhancements and exert a torque on the gas (which has lost its circular motion) in the region between them; this torque is in opposite directions for particles near the inner and outer rings, such that the gas in the vicinity of each ring spirals towards it and falls on it. The gas outer to the outer ring (which has also lost its circular motion due to the tidal torquing) initially expands and then falls back on the outer ring, as it does not acquire escape velocity. Thus both the galaxies get their internal structures enhanced. We have studied the numerical density $(n)$ enhancements of the rings about a fourth of an orbital period after the pericentric approach, and find a substantial density enhancement. We show the results graphically in Figure 1 as a function of the radial distance $r ; n$ and $r$ are normalized with respect to their maximum values in the disk component of the spiral.

We repeated the experiment with two barred spirals, each having a prototypical bar corresponding to a potential $\Phi(r, \theta)=\Phi_{o}\left[1+\Phi_{b}(r) \cos 2 \theta\right]$. In this case the gas exterior to the bar, after the external tidal forcing (which has its maximum effect shortly after the pericentric approach), is torqued into the bar, thus enhancing it. Notice that we have endowed the spirals with a high gaseous content. Elmegreen et al. (1990) find that binary galaxies tend to be both barred and of early type. 
Similar density enhancements of the internal rings and bars of a spiral galaxy occur in the presence of an elliptical perturber (modeled identically as the bulge of the spiral galaxy); but the effect is substantially less on account of the fact that the dynamical friction is lesser in this case (such that the galaxies do not linger much at the pericentric position).

Such effects were noted in the presence of forcing due to a bar (e.g., Simkin et al. 1980; Schwarz 1981; Noguchi 1988) and spirals (e.g., Visser 1980), but actually are typical of galaxies with internal structures, whence due to an external perturbation the gas (lighter content) of the galaxies, exterior to the structures, lose their circular motion; subsequently the torques due to the asymmetric field of the internal structures cause the gas to fall on them, thus enhancing them.

Acknowledgments. It is a matter of great pleasure to thank the organizers of the Colloquium for the excellent hospitality and organization.

\section{References}

Chatterjee, T. K. 1990, Ap\&SS, 163, 127

Chatterjee, T. K. 1992, Ap\&SS, 196, 283

Elmegreen, D. M., Elmegreen, B. G., \& Bellin, D. 1990, ApJ, 364, 415

Noguchi, M. 1988, A\&A, 203, 259

Ostriker, J. P. 1964, ApJ, 140, 1066

Schwarz, M. P. 1981, ApJ, 247, 77

Simkin, S. M., Su, H. J., \& Schwarz, M. P. 1980, ApJ, 273, 404

Visser, H. C. D. 1980, A\&A, 88, 149 